Original Article

\title{
HEALTH-RELATED QUALITY OF LIFE AND DEPRESSION AMONG PATIENTS OF LIVER CIRRHOSIS IN PAKISTAN
}

\author{
MADEEHA MALIK ${ }^{1}$, HAFSA MUSHTAQ ${ }^{2}$, AZHAR HUSSAIN ${ }^{3}$ \\ ${ }_{1,2}^{1,2}$ amdard Institute of Pharmaceutical Sciences, Hamdard University Islamabad, Pakistan, ${ }^{3}$ Dean Faculty of Pharmacy, Hamdard \\ University Islamabad, Pakistan \\ Email: madeehamalik15@gmail.com
}

Received: 05 Nov 2020, Revised and Accepted: 08 Jan 2021

\begin{abstract}
Objective: The present study was designed to assess HRQoL and depression among patients of liver cirrhosis in Pakistan.

Methods: A descriptive cross-sectional study design was used to assess health-related quality of life and depression among liver cirrhosis patients in Pakistan. Two pre-validated questionnaires i.e. SF 36 and HADS were self-administered to a sample of 382 cirrhotic patient's selected using convenience sampling technique for measuring HRQoL and depression, respectively. After data collection, data was cleaned, coded and analyzed using in SPSS version 21.
\end{abstract}

Results: The results highlighted that lowest scores for HRQoL were observed in the domain of role physical $(40.02, \pm 24.01)$ followed by role emotional $(41.93, \pm 26.99)$ whereas the highest scores were observed in the domain of physical functioning $(51.87, \pm 15.22)$.

Conclusion: The results of the present study concluded that liver cirrhosis patients in Pakistan had poor HRQoL across all domains along with mild to moderate depression. While all domains were affected, the greatest impact was on role limitation due to physical as well as emotional problems. The most affected group among patients were females and patients having age greater than $60 \mathrm{y}$. Besides this severity and advanced stage of cirrhosis had a negative effect on all domains of HRQoL. Presence of complications further reduced HRQoL and increased depressive symptoms among liver cirrhosis patients.

Keywords: Liver cirrhosis, Health-related quality of life, Depression, Liver disease stages, SF 36, HADS, Pakistan

(C) 2021 The Authors. Published by Innovare Academic Sciences Pvt Ltd. This is an open access article under the CC BY license (https://creativecommons.org/licenses/by/4.0/) DOI: https://dx.doi.org/10.22159/ijcpr.2021v13i2.41546. Journal homepage: https://innovareacademics.in/journals/index.php/ijcpr

\section{INTRODUCTION}

Chronic liver disorders are becoming the most important public health issue across the globe contributing substantially to noncommunicable disease burden in developed as well as developing countries. More than 500 million patients have been diagnosed with chronic liver disease caused due to a viral cause. On the other hand, the prevalence of chronic liver disorders due to non-viral etiology such as non-alcoholic and alcoholic fatty liver disease, cryptogenic hepatitis and autoimmune hepatitis is also rising significantly [1] Liver cirrhosis is a key cause of increased mortality and morbidity among chronic liver disorders. The death rate due to liver cirrhosis has increased to more than one million people in the year 2010 as compared to 0.6 million deaths in year 1980 [2]. This rising prevalence can be linked to an increased burden of risk factors. Infection with hepatitis virus $\mathrm{B}$ and $\mathrm{C}$ are the main contributing factors towards liver cirrhosis, while intake of alcohol and other chronic diseases for instance diabetes mellitus, have proven to be main risk factors for cirrhosis in developed countries [3].

Liver cirrhosis has not only increased the mortality rate but has negative impact on the health-related quality of life (HRQoL) of the patients. Patients diagnosed with liver cirrhosis suffer from symptoms including extreme fatigue, pruritus, depression and cirrhotic complications such as ascites, hepatic encephalopathy, recurrent variceal bleeding and spontaneous bacterial peritonitis. The presence of these complications mainly leads to a decline in health-related quality of life and well-being of patients [4]. The decline in HRQoL and mental health is present throughout the course of disease but is more profound in advanced stages of cirrhosis. Patients having cirrhosis tend to have poor HRQoL due to the presence of muscle cramps and pruritus [5]. Moreover, half of the patients diagnosed with cirrhosis commonly have depression. The well-being of diagnosed patients is highly affected due to these complications, which is not clearly assessed by using clinical disease markers such as Child-Pugh Classification [6]. Assessment of HRQoL and depression are considered as efficient tools for monitoring patient care. It focuses on effects of disease on emotional, physical and mental functioning which could help in evaluating the wide range of problems which cirrhotic patients can face. Apart from clinical endpoints, patient reported outcomes such as HRQoL should be the prime focus for healthcare professionals in order to improve disease prognosis [4].

Cirrhosis has been identified as common cause of chronic liver disorder in Pakistan. Pakistan is among the ten countries having highest burden of liver diseases like hepatitis which can lead towards cirrhosis [7]. A study conducted in gastroenterology clinics revealed that health-related quality of life was lower in patients with liver cirrhosis. Factors such as hemoglobin, albumin and decompensated liver cirrhosis were linked with poor HRQoL [8]. A study conducted in Islamabad reported that the majority of patients with chronic liver disease were diagnosed with hepatitis. Patients reported poor healthrelated quality of life [9]. Health related quality of life was also reported to be low in hepatitis B patients. Less educated patients, patients in advanced stages of disease, elderly and female patients had relatively poor HRQoL [10]. Decline in health-related quality of life in liver cirrhosis patients leads to poor treatment outcomes, reduced compliance to treatment, increased complications and poor prognosis of liver cirrhosis. Limited research has focused on assessment of HRQoL and depression in liver cirrhosis patients in Pakistan. Extensive research is required to assess current scenario and factors affecting HRQoL and depression among patients of liver cirrhosis in Pakistan. Thus, the present study was designed to assess HRQoL and depression among patients of liver cirrhosis in Pakistan.

\section{MATERIALS AND METHODS}

A descriptive cross-sectional study design was used to assess healthrelated quality of life and depression among liver cirrhosis patients attending healthcare facilities located in twin cities (Islamabad and Rawalpindi) of Pakistan. Study approval was taken from the Ethical 
Committee of Hamdard University (BASR-81-82). All the public and private health care facilities treating liver cirrhosis patients located in twin cities were included in the study. Patients diagnosed with liver cirrhosis with ages between 18-60 y were the study respondents. However, patients who did not fall in Child-Pugh score criteria for liver cirrhosis and those having co-morbidities not affecting the current condition were excluded from the study. Approval was also taken from Medical superintendents of different healthcare facilities of Rawalpindi and Islamabad. Patients were briefed regarding the nature and objectives of the study. Verbal and written consent was obtained prior to data collection. Respondents were ensured of the confidentiality of their responses along with full right to withdraw from the study at any time. The sample size was calculated by using Rao soft at 95\% confidence interval and 5\% margin of error which came to be 382. Convenience sampling technique was used for the selection of respondents available and willing to participate at the time of data collection. Two pre validated questionnaires i.e. SF 36 and HADS for assessment of HRQoL and Depression, respectively, were used. The questionnaires were self-administered and collected back on the same day to avoid biasness. SF-36 consist of eight subscales including perceived mental health (5 items), physical functioning (10 items), general health perceptions ( 5 items), role limitations due to physical problems ( 4 items), role limitation due to emotional problems (3 items), social functioning ( 2 items), vitality ( 4 items) and bodily pain (2 items) [11] while Hospital Anxiety and Depression Scale (HADS) consists of 7 items each for anxiety and depression [12]. Pilot testing was performed on $10 \%$ of the sample size for assessing the reliability of the tools. The value of Cronbach's alpha for SF 36 was 0.73 and 0.69 for Hospital Anxiety and Depression Scale (HADS). After data collection, data was cleaned, coded and analyzed using SPSS version 21. Descriptive statistics comprising of frequency and percentages were calculated. Nonparametric tests Kruskal Wallis and Mann Whitney tests ( $\mathrm{p} \geq 0.0$ E) applied to find out the differences among different variables.

\section{RESULTS}

\section{Demographic characteristics of respondents}

Out of 382 respondents, $58.6 \%(n=224)$ were males and $41.4 \%$ $(n=158)$ were females. Nearly, 67.3\% $(n=257)$ resided in rural settings and $32.7 \%(n=125)$ were from urban areas. The patients were suffering from different stages of liver cirrhosis: stage I $(\mathrm{n}=184,48.2 \%)$, stage-II $(\mathrm{n}=143,37.4 \%)$, stage-III $(\mathrm{n}=43,13.4 \%)$ and stage-IV ( $\mathrm{n}=4,1 \%$ ). Regarding the type of treatment, 95\% $(n=363)$ were being treated by medicines and only $1.3 \%(n=5)$ had undergone liver transplantt. Furthermore, 53.1\% $(n=203)$ were suffering from hypertension and $30.9 \%(n=118)$ were previously diagnosed with diabetes mellitus (table 1).

Table 1: Demographic characteristics of respondents

\begin{tabular}{|c|c|c|}
\hline Indicators & & Total n (\%) \\
\hline \multirow[t]{5}{*}{ Age } & $18-30 \mathrm{y}$ & $120(31.4)$ \\
\hline & $31-40 y$ & $194(50.8)$ \\
\hline & $41-50 y$ & $45(11.8)$ \\
\hline & $51-60 y$ & $16(4.2)$ \\
\hline & $>60 y$ & $7(1.8)$ \\
\hline \multirow[t]{2}{*}{ Gender } & Males & $224(58.6)$ \\
\hline & Females & $158(41.4)$ \\
\hline \multirow[t]{2}{*}{ Marital Status } & Married & $298(78)$ \\
\hline & Unmarried & $84(22)$ \\
\hline \multirow[t]{6}{*}{ Level of Education } & Illiterate & $94(24.6)$ \\
\hline & Primary & $145(38)$ \\
\hline & Secondary & $100(26.2)$ \\
\hline & Bachelors & $28(7.3)$ \\
\hline & Masters & $14(3.7)$ \\
\hline & Post-Graduate & $1(0.3)$ \\
\hline \multirow[t]{6}{*}{ Income Level } & $\leq 20,000$ & $262(68.6)$ \\
\hline & $21,000-40,000$ & $92(24.1)$ \\
\hline & $41,000-60,000$ & $24(6.3)$ \\
\hline & $61,000-80,000$ & $4(1.0)$ \\
\hline & $81,000-100,000$ & $0(0)$ \\
\hline & $>100,000$ & $0(0)$ \\
\hline \multirow[t]{2}{*}{ Setting } & Urban & $125(32.7)$ \\
\hline & Rural & $257(67.3)$ \\
\hline \multirow[t]{4}{*}{ Stage of Liver Cirrhosis } & Stage-1 & $184(48.2)$ \\
\hline & Stage-2 & $143(37.4)$ \\
\hline & Stage-3 & $51(13.4)$ \\
\hline & Stage-4 & $4(1.0)$ \\
\hline \multirow[t]{4}{*}{ Type of Co-morbidity } & None & $56(14.7)$ \\
\hline & Hypertension & $203(53.1)$ \\
\hline & Diabetes & $118(30.9)$ \\
\hline & Others & $5(1.3)$ \\
\hline \multirow[t]{4}{*}{ Type of Treatment } & Medicines & $363(95.0)$ \\
\hline & Transplant & $5(1.3)$ \\
\hline & None & $14(3.7)$ \\
\hline & Other & $0(0)$ \\
\hline \multirow[t]{3}{*}{ Mode of Treatment Payment } & Self-finance & $52(13.6)$ \\
\hline & Free & $328(85.9)$ \\
\hline & Insurance & $2(0.5)$ \\
\hline \multirow[t]{4}{*}{ Symptoms and Signs affecting HRQoL } & Cognitive dysfunction & $1(0.3)$ \\
\hline & Insomnia & $102(26.7)$ \\
\hline & Fatigue & $270(70.7)$ \\
\hline & Others & $9(2.4)$ \\
\hline
\end{tabular}


Table 2 Domains of health related quality of life among liver cirrhosis patients in Pakistan

\begin{tabular}{|c|c|c|c|}
\hline Indicator & Mean & Median & Standard deviation \\
\hline Physical functioning & 51.87 & 50.00 & \pm 15.22 \\
\hline Role physical & 40.02 & 50.00 & \pm 24.01 \\
\hline Bodily pain & 45.99 & 45.00 & \pm 15.88 \\
\hline General health & 42.29 & 45.00 & \pm 10.66 \\
\hline Social functioning & 50.16 & 45.00 & \pm 14.62 \\
\hline Role emotional & 41.93 & 33.33 & \pm 26.99 \\
\hline Vitality & 45.74 & 48.00 & \pm 11.84 \\
\hline Mental health & 47.09 & 48.00 & \pm 10.57 \\
\hline
\end{tabular}

Domains of health-related quality of life among liver cirrhosis patients in Pakistan

The results highlighted that lowest scores for HRQoL were observed in the domain of role physical $(40.02, \pm 24.01)$ followed by role emotional $(41.93, \pm 26.99)$ whereas highest scores were observed in the domain of physical functioning $(51.87, \pm 15.22)$. A detailed description is given (table 2 ).

Comparison of health-related quality of life domains by demographic variables among liver cirrhosis patients in Pakistan

Significant difference $(\mathrm{p} \leq 0.05)$ was found in HRQoL domain among different age groups, income level, stage of cirrhosis and type of comorbidity. Elder patients had poor HRQoL with a significant decline in physical and mental health. Respondents having income between Rs. 41,000-60,000 reported poor HRQoL. Respondents having stage IV cirrhosis reported poor health-related quality of life in both physical and mental health domains. Respondents having diabetes as co-morbidity along with cirrhosis had poor health-related quality of life (table 3).

Assessment of depression among liver cirrhosis patients in pakistan among different demographic variables

The results of the current study showed that respondents having age 51-60 y $(10.43, \pm 2.85)$ were more depressed as compared to respondents having age between $31-40$ y $(9.09, \pm 2.40)$. Females respondents were more depressed $(9.63, \pm 2.66)$ as compared to male respondents $(9.00, \pm 2.12)$ having liver cirrhosis. Respondents having stage 4 of liver cirrhosis were more depressed $(12.00, \pm 4.61)$ as compared to those diagnosed with stage 1 of liver cirrhosis $(9.06, \pm 2.35)$. Respondents not undergoing any sort of treatment were more depressed $(10.50, \pm 2.90)$ as compared to those using medicines for management $(9.21, \pm 2.34)$. Respondents having cognitive dysfunction were more depressed $(15.00, \pm 0.01)$ as compared to those having fatigue only $(9.24, \pm 2.19)$. A detailed description is given (table 4).

Table 3: Comparison of health-related quality of life domains by demographic variables among liver cirrhosis patients in Pakistan

\begin{tabular}{|c|c|c|c|c|c|c|c|c|c|c|c|c|c|}
\hline \multirow[t]{2}{*}{ Indicators } & & \multicolumn{4}{|c|}{ Physical health component score } & \multicolumn{4}{|c|}{ Mental ealth component score } & \multicolumn{4}{|c|}{ Composite score } \\
\hline & & n & $\begin{array}{l}\text { Mean } \\
\text { ranks }\end{array}$ & $\begin{array}{l}\text { Test } \\
\text { statistics }\end{array}$ & $\begin{array}{l}\text { p- } \\
\text { value }\end{array}$ & n & $\begin{array}{l}\text { Mean } \\
\text { ranks }\end{array}$ & $\begin{array}{l}\text { Test } \\
\text { statistics }\end{array}$ & $\begin{array}{l}\text { p- } \\
\text { value }\end{array}$ & n & $\begin{array}{l}\text { Mean } \\
\text { ranks }\end{array}$ & $\begin{array}{l}\text { Test } \\
\text { statistics }\end{array}$ & $\begin{array}{l}\text { p- } \\
\text { value }\end{array}$ \\
\hline \multirow[t]{5}{*}{ Age } & $18-30 y$ & 120 & 198.77 & $34.309 \mathrm{~b}$ & 0.001 & 120 & 195.18 & $31.324 \mathrm{~b}$ & 0.001 & 120 & 196.29 & $35.056^{b}$ & 0.001 \\
\hline & $31-40 y$ & 194 & 204.80 & & & 194 & 203.20 & & & 194 & 204.22 & & \\
\hline & $41-50 y$ & 45 & 144.49 & & & 45 & 166.93 & & & 45 & 155.67 & & \\
\hline & $51-60 y$ & 16 & 103.41 & & & 16 & 71.66 & & & 16 & 87.97 & & \\
\hline & $>60 y$ & 7 & 47.86 & & & 7 & 79.14 & & & 7 & 43.43 & & \\
\hline \multirow[t]{2}{*}{ Gender } & Males & 224 & 193.02 & $16089.500^{a}$ & 0.332 & 224 & 189.13 & $16955.000^{a}$ & 0.893 & 224 & 190.01 & $16573.000^{a}$ & 0.668 \\
\hline & Females & 158 & 181.98 & & & 158 & 187.60 & & & 158 & 185.12 & & \\
\hline Marital & Married & 298 & 184.40 & $10847.000^{a}$ & 0.165 & 298 & 183.76 & $10661.000^{\mathrm{a}}$ & 0.110 & 298 & 183.93 & $10821.000^{\mathrm{a}}$ & 0.169 \\
\hline Status & Unmarried & 84 & 203.21 & & & 84 & 205.48 & & & 84 & 202.54 & & \\
\hline Level of & Illiterate & 94 & 152.20 & $15.770^{\mathrm{b}}$ & 0.232 & 94 & 157.14 & $5.985^{b}$ & 0.150 & 94 & 155.91 & $13.872^{\mathrm{b}}$ & 0.628 \\
\hline \multirow{4}{*}{ Education } & Primary & 145 & 190.48 & & & 145 & 186.94 & & & 145 & 186.36 & & \\
\hline & Secondary & 100 & 204.05 & & & 100 & 201.49 & & & 100 & 205.25 & & \\
\hline & Bachelors & 28 & 221.43 & & & 28 & 206.09 & & & 28 & 218.54 & & \\
\hline & Masters & 14 & 217.21 & & & 14 & 204.18 & & & 14 & 219.04 & & \\
\hline Income & $\leq 20,000$ & 262 & 199.49 & $21.362^{\mathrm{b}}$ & 0.001 & 262 & 198.04 & $8.327 \mathrm{~b}$ & 0.040 & 262 & 198.79 & $19.211^{b}$ & 0.001 \\
\hline \multirow[t]{3}{*}{ Level } & $21,000-40,000$ & 92 & 162.51 & & & 92 & 171.91 & & & 92 & 164.68 & & \\
\hline & $41,000-60,000$ & 24 & 139.85 & & & 24 & 143.48 & & & 24 & 134.91 & & \\
\hline & $61,000-80,000$ & 4 & 350.50 & & & 4 & 216.00 & & & 4 & 330.75 & & \\
\hline \multirow[t]{2}{*}{ Setting } & Urban & 125 & 211.57 & $12721.000^{a}$ & 0.004 & 125 & 198.23 & $14363.000^{a}$ & 0.226 & 125 & 209.95 & $12798.500^{a}$ & 0.006 \\
\hline & Rural & 257 & 177.28 & & & 257 & 183.77 & & & 257 & 177.29 & & \\
\hline Stage of & Stage-1 & 184 & 186.79 & $8.754^{b}$ & 0.033 & 184 & 181.91 & $11.085^{b}$ & 0.011 & 184 & 181.46 & $11.553^{\mathrm{b}}$ & 0.009 \\
\hline Liver & Stage-2 & 143 & 194.06 & & & 143 & 199.91 & & & 143 & 197.29 & & \\
\hline \multirow[t]{2}{*}{ Cirrhosis } & Stage-3 & 51 & 191.39 & & & 51 & 192.54 & & & 51 & 198.65 & & \\
\hline & Stage-4 & 4 & 32.00 & & & 4 & 27.50 & & & 4 & 22.00 & & \\
\hline \multirow{4}{*}{$\begin{array}{l}\text { Type of Co- } \\
\text { morbidity }\end{array}$} & None & 56 & 214.21 & $19.171^{\mathrm{b}}$ & 0.001 & 56 & 196.68 & $8.297 \mathrm{~b}$ & 0.040 & 56 & 213.07 & $15.658^{\mathrm{b}}$ & 0.001 \\
\hline & Hypertension & 203 & 197.98 & & & 203 & 192.93 & & & 203 & 193.25 & & \\
\hline & Diabetes & 118 & 165.07 & & & 118 & 182.36 & & & 118 & 173.04 & & \\
\hline & Others & 5 & 40.50 & & & 5 & 57.30 & & & 5 & 33.90 & & \\
\hline Type of & Medicines & 363 & 189.15 & $2.196^{b}$ & 0.333 & 363 & 189.88 & $1.320^{\mathrm{b}}$ & 0.517 & 363 & 188.92 & $1.222^{\mathrm{b}}$ & 0.543 \\
\hline \multirow[t]{2}{*}{ Treatment } & Transplant & 5 & 118.10 & & & 5 & 145.00 & & & 5 & 135.60 & & \\
\hline & None & 14 & 196.89 & & & 14 & 168.79 & & & 14 & 183.21 & & \\
\hline Mode of & Self-finance & 52 & 70.70 & $70.566^{a}$ & 0.001 & 52 & 76.41 & $63.786^{a}$ & 0.001 & 52 & 65.67 & $76.518^{a}$ & 0.001 \\
\hline \multicolumn{14}{|l|}{ Payment } \\
\hline $\begin{array}{l}\text { Symptoms } \\
\text { and Signs }\end{array}$ & $\begin{array}{l}\text { Cognitive } \\
\text { dysfunction }\end{array}$ & 1 & 1.50 & $19.162^{b}$ & 0.001 & 1 & 7.50 & $23.884 b$ & 0.001 & 1 & 2.50 & $26.120^{\mathrm{b}}$ & 0.001 \\
\hline affecting & Insomnia & 102 & 183.48 & & & 102 & 151.33 & & & 102 & 167.82 & & \\
\hline \multirow[t]{2}{*}{ HRQoL } & Fatigue & 270 & 195.85 & & & 270 & 203.39 & & & 270 & 201.30 & & \\
\hline & Others & 9 & 49.33 & & & 9 & 75.00 & & & 9 & 44.78 & & \\
\hline
\end{tabular}

Mann-Whitney, Kruskal Wallis ${ }^{\mathrm{b}}(\mathrm{p} \geq 0.05)$ 
Malik et al.

Table 4: Assessment of depression among liver cirrhosis patients in Pakistan among different demographic variables

\begin{tabular}{|c|c|c|c|}
\hline Indicators & & Total mean $( \pm S D)$ & Severity of depression \\
\hline \multirow{5}{*}{ Age } & $18-30 \mathrm{y}$ & $9.11( \pm 2.05)$ & Mild \\
\hline & $31-40 y$ & $9.09( \pm 2.40)$ & mild \\
\hline & $41-50 \mathrm{y}$ & $10.04( \pm 2.50)$ & Mild \\
\hline & $51-60 y$ & $10.43( \pm 2.85)$ & Mild \\
\hline & $>60 y$ & $8.85( \pm 3.76)$ & Mild \\
\hline \multirow[t]{2}{*}{ Gender } & Males & $9.00( \pm 2.12)$ & Mild \\
\hline & Females & $9.63( \pm 2.66)$ & Mild \\
\hline \multirow[t]{2}{*}{ Marital Status } & Married & $9.30( \pm 2.51)$ & Mild \\
\hline & Unmarried & $9.11( \pm 1.79)$ & Mild \\
\hline \multirow[t]{6}{*}{ Level of Education } & Illiterate & $9.82( \pm 2.40)$ & Mild \\
\hline & Primary & $8.90( \pm 2.22)$ & Mild \\
\hline & Secondary & $9.09( \pm 2.29)$ & Mild \\
\hline & Bachelors & $10.28( \pm 2.97)$ & Mild \\
\hline & Masters & $8.61( \pm 1.89)$ & Mild \\
\hline & Post-Graduate & $7.00( \pm 0.01)$ & Normal \\
\hline \multirow[t]{4}{*}{ Income Level } & $\leq 20,000$ & $9.01( \pm 2.16)$ & Mild \\
\hline & $21,000-40,000$ & $9.62( \pm 2.36)$ & Mild \\
\hline & $41,000-60,000$ & $10.69( \pm 3.93)$ & Mild \\
\hline & $61,000-80,000$ & $9.00( \pm 0.01)$ & Mild \\
\hline \multirow[t]{2}{*}{ Setting } & Urban & $9.31( \pm 2.45)$ & Mild \\
\hline & Rural & $9.24( \pm 2.34)$ & Mild \\
\hline \multirow[t]{4}{*}{ Stage of Liver Cirrhosis } & Stage-1 & $9.06( \pm 2.35)$ & Mild \\
\hline & Stage-2 & $9.09( \pm 2.01)$ & Mild \\
\hline & Stage-3 & $10.27( \pm 2.85)$ & Mild \\
\hline & Stage-4 & $12.00( \pm 4.61)$ & Moderate \\
\hline \multirow[t]{4}{*}{ Type of Co-morbidity } & None & $9.85( \pm 2.78)$ & Mild \\
\hline & Hypertension & $8.99( \pm 2.36)$ & Mild \\
\hline & Diabetes & $9.38( \pm 1.98)$ & Mild \\
\hline & Others & $11.00( \pm 4.58)$ & Moderate \\
\hline \multirow[t]{3}{*}{ Type of Treatment } & Medicines & $9.21( \pm 2.34)$ & Mild \\
\hline & Transplant & $9.40( \pm 2.60)$ & Mild \\
\hline & None & $10.50( \pm 2.90)$ & Mild \\
\hline \multirow[t]{2}{*}{ Mode of Treatment Payment } & Self-finance & $11.01( \pm 3.62)$ & Moderate \\
\hline & Free & $8.98( \pm 1.98)$ & Mild \\
\hline \multirow[t]{4}{*}{ Symptoms and Signs affecting HRQoL } & Cognitive dysfunction & $15.00( \pm 0.01)$ & Severe \\
\hline & Insomnia & $9.38( \pm 2.76)$ & Mild \\
\hline & Fatigue & $9.24( \pm 2.19)$ & Mild \\
\hline & Others & $8.00( \pm 2.12)$ & Mild \\
\hline
\end{tabular}

Table 5: Comparison of depression among liver cirrhosis patients in Pakistan among different demographic variables

\begin{tabular}{|c|c|c|c|c|c|}
\hline Indicators & & n & Mean ranks & Test statistics & p-value \\
\hline & $18-30 y$ & 120 & 184.85 & & \\
\hline \multirow[t]{4}{*}{ Age } & $31-40 y$ & 194 & 184.33 & $9.544^{b}$ & 0.049 \\
\hline & $41-50 y$ & 45 & 224.81 & & \\
\hline & $51-60 y$ & 16 & 239.50 & & \\
\hline & $>60 y$ & 7 & 152.14 & & \\
\hline \multirow[t]{2}{*}{ Gender } & Males & 224 & 184.27 & $16077.500^{\mathrm{a}}$ & 0.149 \\
\hline & Females & 158 & 200.60 & & \\
\hline \multirow[t]{2}{*}{ Marital Status } & Married & 298 & 193.00 & $11880.500^{\mathrm{a}}$ & 0.500 \\
\hline & Unmarried & 84 & 183.93 & & \\
\hline \multirow[t]{6}{*}{ Level of Education } & Illiterate & 94 & 217.60 & & \\
\hline & Primary & 145 & 177.91 & $13.528^{\mathrm{b}}$ & 0.019 \\
\hline & Secondary & 100 & 180.16 & & \\
\hline & Bachelors & 28 & 223.89 & & \\
\hline & Masters & 14 & 168.00 & & \\
\hline & Post-Graduate & 1 & 51.00 & & \\
\hline \multirow[t]{4}{*}{ Income Level } & $\leq 20,000$ & 262 & 183.40 & & \\
\hline & $21,000-40,000$ & 92 & 203.76 & $5.160^{\mathrm{b}}$ & 0.160 \\
\hline & $41,000-60,000$ & 24 & 227.35 & & \\
\hline & $61,000-80,000$ & 4 & 181.50 & & \\
\hline \multirow[t]{2}{*}{ Setting } & Urban & 125 & 191.63 & $15856.500^{\mathrm{a}}$ & 0.938 \\
\hline & Rural & 257 & 190.70 & & \\
\hline \multirow[t]{4}{*}{ Stage of Liver Cirrhosis } & Stage-1 & 184 & 185.86 & & \\
\hline & Stage-2 & 143 & 180.89 & $10.420^{\mathrm{b}}$ & 0.015 \\
\hline & Stage-3 & 51 & 233.97 & & \\
\hline & Stage- 4 & 4 & 239.75 & & \\
\hline \multirow[t]{4}{*}{ Type of Co-morbidity } & None & 56 & 208.14 & & \\
\hline & Hypertension & 203 & 177.69 & $6.548^{\mathrm{b}}$ & 0.088 \\
\hline & Diabetes & 118 & 205.42 & & \\
\hline & Others & 5 & 202.00 & & \\
\hline
\end{tabular}




\begin{tabular}{|c|c|c|c|c|c|}
\hline \multirow[t]{3}{*}{ Type of Treatment } & Medicines & 363 & 188.73 & \multirow{3}{*}{$3.792^{\mathrm{b}}$} & \multirow{3}{*}{0.150} \\
\hline & Transplant & 5 & 201.40 & & \\
\hline & None & 14 & 246.04 & & \\
\hline Mode of Treatment & Self-finance & 52 & 243.25 & \multirow[t]{2}{*}{$5733.000^{\mathrm{a}}$} & \multirow[t]{2}{*}{0.001} \\
\hline Payment & Free & 328 & 181.53 & & \\
\hline Symptoms and Signs & Cognitive dysfunction & 1 & 369.50 & \multirow[t]{4}{*}{$5.962^{\mathrm{b}}$} & \multirow[t]{4}{*}{0.113} \\
\hline \multirow[t]{3}{*}{ affecting HRQoL } & Insomnia & 102 & 185.46 & & \\
\hline & Fatigue & 270 & 194.44 & & \\
\hline & Others & 9 & 131.11 & & \\
\hline
\end{tabular}

Mann-Whitney ${ }^{a}$,Kruskal Wallis ${ }^{b}(p \geq 0.05)$

\section{Comparison of depression among liver cirrhosis patients in Pakistan among different demographic variables}

Mann-Whitney and Kruskal Wallis tests were used to find the differences among different demographic variables. Significant difference $(p \leqq 0.05)$ was found in depression among respondents having different age groups $(\mathrm{p}=0.049)$, level of education $(\mathrm{p}=0.019)$, stage of cirrhosis $(\mathrm{p}=0.015)$ and mode of payment for treatment $(p=0.001)$. Respondents having age between 51-60 y were more depressed as compared to younger respondents having age between 31-40 y. Respondents having bachelors as their education were more depressed as compared to those having post-graduate education. Respondents having stage 4 cirrhosis were more depressed as compared to those diagnosed with stage 1 and 2 liver cirrhosis. Respondents undergoing treatment through self-finance were more depressed as compared to those undergoing free treatment. No significant difference $(\mathrm{p} \geqq 0.05)$ was found in depression among respondents having different genders, marital status, income level, setting, type of co-morbidity, type of treatment and symptoms of cirrhosis affecting HRQoL (table 5).

\section{DISCUSSION}

The increasing prevalence of liver cirrhosis in developed as well as developing countries along with increased morbidity and mortality has highlighted it as the most important global public health issue. The severity and progressive nature of the disease has a significant negative impact on health related quality of life of patients. The complications due to liver cirrhosis further complicate treatment outcomes and leads to poor patient reported outcomes [13]. The findings of the present study reported poor HRQoL among liver cirrhosis patients and highlighted a significant impact of liver cirrhosis on various domains of health-related quality of life. High scores of health-related quality of life were observed for physical functioning and social functioning, whereas low scores were observed for role limitation due to physical and emotional functioning followed by lower scores in general health. Similar results were observed in a study conducted in USA where majority of the patients reported decline in physical and emotional health due to liver cirrhosis [14].

The debilitating symptoms, as well as severe complications of liver cirrhosis, can severely affect the physical activities of patients. The findings of the current research revealed that most of the liver cirrhosis patients had difficulty in carrying out strenuous activities such as running, lifting heavy items and walking more than a mile. Majority of the patients achieved less than they had thought they could have achieved. Similar results were reported in a study conducted in Austria where physical activities of liver cirrhosis patients were reported compromised [15]. The findings of the current research showed that most of the liver cirrhosis patients had less energy and felt tired to perform daily activities. Patients reported body pain most of the times, which interfered with their daily activities. Similar results were reported in a study conducted in Poland where patients had low energy levels and had fatigue as major symptom of liver disease [16]. The results of the present study showed that the social functioning of most of the liver cirrhosis patients was moderately impaired. Liver cirrhosis patients were unable to give proper time to their work and performed less activities than usual due to emotional disturbances. Similar results were obtained from a study conducted in USA where patients with cirrhosis due to hepatitis reported poor social functioning [17].
Physical, as well as mental health can be impaired with patients diagnosed with liver cirrhosis. The results of the present study highlighted that patients aged $60 \mathrm{y}$ or above had impaired health related quality of life across all domains. This might be due to the fact that elderly patients face severe complications of cirrhosis and have poor prognosis. Similar results were obtained from studies conducted in Europe where elder patients were reported with declined HRQoL [18]. The findings of the current research highlighted that females had relatively poor HRQoL in physical as well as mental health domains as compared to male liver cirrhosis patients. This might be due to the fact that females have low physical strength and are more emotional related to health as compared to males. A study conducted in Brazil reported similar results with females having poor HRQoL across all domains [13]. Moreover, the present study reported that patients having income level between Rs. 41000-60000 had poor health-related quality of life across all domains. This might be due to the fact that middle-class income patients were unable to avail free treatments through the government and had to pay out of their pockets. Furthermore, patients presenting with stage IV cirrhosis had poor health-related quality of life. However, patients with stage I of liver cirrhosis also reported poor health-related quality of life as compared to stage II and III patients. This might be due to the fact that limited knowledge about the disease and its prognosis as well as reduced social support in patients with stage I can lead to decreased HRQoL. Similar results were found from a study conducted in India where poor health-related quality of life was reported in advanced stages of disease [19].

Liver cirrhosis can lead to major psychological distress in diagnosed individuals causing moderate to severe depression. Due to prolonged therapy and poor prognosis, decline in mental functioning is common among patients of liver cirrhosis. The presence of depressive symptoms can lead to reduced quality of life, adherence to treatment and increased functional impairment [20]. The results of the current study revealed that majority of the liver cirrhosis patients had mild to moderate depression. Most of them felt tensed, restless and frightened quite often and had little interest in their appearance or doing routine activities. Similar results were reported in studies conducted in USA where majority of the cirrhotic patients had depressive symptoms [21,22]. Moreover, results of the present study showed that elder cirrhotic patients were relatively more depressed than younger patients. This might be due to the fact that older patients had more debilitating symptoms and complications, poor prognosis and less chances of survival. A study conducted in Taiwan reported similar results with older patients having higher level of depression as compared to younger patients [23].

Patients having depression with cirrhotic liver disorders have poor clinical outcomes and decline in mental health. The results of the current study revealed that female cirrhotic patients were more depressed than male counterparts. This might be due to the fact that as females have more family responsibilities to fulfill so depression prevails in this gender. Similarly, married respondents had more depressive symptoms as compared to unmarried. Similar results were reported in a study conducted in Europe where females and married patients had a higher level of depression [24]. Moreover, the current study reported that literate patients were found more depressed as compared to illiterate ones. This might be due to the fact that educated patients could better understand the disease and might be depressed due to its complications and prognosis. Similar results were observed in a study conducted in Brazil where educated cirrhotic patients had higher level of depression [25]. 
The development and prognosis of liver cirrhosis is influenced by psychological factors such as presence of depressive symptoms which can lead to an adverse impact on the treatment of disease. The results of the current study highlighted that patients with stage IV liver cirrhosis were more depressed as compared to those diagnosed with stage I of cirrhosis. Patients not undergoing any sort of treatment had more depressive symptoms than patients undergoing pharmacological management or liver transplant. This might be due to the fact that in advanced stages of disease the complications were more severe and management of patient was difficult. A study conducted in China reported similar results with higher level of depression in advanced stages of the liver disease [26].

Assessment and treatment of psychological disorders is necessary for effective management of liver cirrhosis. The current study highlighted that cirrhotic patients with cognitive dysfunction and fatigue as a symptom of liver cirrhosis were found to be more depressed than others. This might be due to the fact that these symptoms interfered with the normal functioning of individuals diagnosed with liver cirrhosis. Similar results were reported from studies conducted in USA where a negative impact was observed on patient mental health due to the symptoms and complications of cirrhosis $[20,22]$.

\section{CONCLUSION}

The results of the present study concluded that liver cirrhosis patients in Pakistan had poor HRQoL across all domains along with mild to moderate depression. While all domains were affected, the greatest impact was on role limitation due to physical as well as emotional problems. The most affected group among patients were females and patients having age greater than $60 \mathrm{y}$. Besides this, severity and advanced stage of cirrhosis had negative effect on all domains of HRQoL. Presence of complications further reduced HRQoL and increased depressive symptoms among liver cirrhosis patients. All relevant stakeholders must collaborate to design innovative pharmacotherapy strategies for improving health-related quality of life and depression in liver cirrhosis. Well-structured pharmacotherapy and psychosocial support programs should be designed so the management of liver cirrhosis could be made more optimal and clinical outcomes can be achieved timely with which prognosis of disease can be improved.

\section{FUNDING}

Nil

\section{AUTHORS CONTRIBUTIONS}

All the authors have contributed equally.

\section{CONFLICTS OF INTERESTS}

\section{Declared none}

\section{REFERENCES}

1. Wong MC, Huang J. The growing burden of liver cirrhosis: implications for preventive measures. Springer; 2018.

2. Setiawan VW, Stram DO, Porcel J, Lu SC, Le Marchand L, Noureddin M. Prevalence of chronic liver disease and cirrhosis by underlying cause in understudied ethnic groups: the multiethnic cohort. Hepatology 2016;64:1969-77.

3. Wiegand J, Berg T. The etiology, diagnosis and prevention of liver cirrhosis: part 1 of a series on liver cirrhosis. Deutsches Arzteblatt International 2013;110:85.

4. Younossi ZM, Boparai N, Price LL, Kiwi ML, McCormick M, Guyatt G. Health-related quality of life in chronic liver disease: the impact of type and severity of the disease. Am J Gastroenterol 2001;96:2199-205.

5. Janani K, Jain M, Vargese J, Srinivasan V, Harika K, Michael T, et al. Health-related quality of life in liver cirrhosis patients using SF-36 and CLDQ questionnaires. Clin Exp Hepatol 2018;4:232.

6. Inglis SC, Clark RA, McAlister FA, Stewart S, Cleland JG. Which components of heart failure programmes are effective? A systematic review and maalysis of the outcomes of structured telephone support or telemonitoring as the primary component of chronic heart failure management in 8323 patients: abridged Cochrane review. Eur J Heart Failure 2011;13:1028-40.

7. Butt AA, Yan P, Bonilla H, Abou Samra AB, Shaikh OS, Simon TG, et al. Effect of addition of statins to antiviral therapy in hepatitis C virus-infected persons: results from ERCHIVES. Hepatology 2015;62:365-74.

8. Parkash O, Iqbal R, Jafri F, Azam I, Jafri W. Frequency of poor quality of life and predictors of health related quality of life in cirrhosis at a tertiary care hospital Pakistan. BMC Res Notes 2012;5:446.

9. Atiq M, Gill M, Khokhar N. Quality of life assessment in Pakistani patients with chronic liver disease. Emotion 2004;5:0.05.

10. Ul Haq N, Hassali MA, Shafie AA, Saleem F, Aljadhey H. A cross sectional assessment of health related quality of life among patients with Hepatitis-B in Pakistan. Health Quality Life Outcomes 2012;10:91.

11. Pulgar A, Alcala A, Reyes del Paso GA. Psychosocial predictors of quality of life in hematological cancer. Behavioral Med 2015;41:1-8.

12. Oberoi D, White V, Seymour J, Prince HM, Harrison S, Jefford M, et al. The course of anxiety, depression and unmet needs in survivors of diffuse large B cell lymphoma and multiple myeloma in the early survivorship period. J Cancer Survivorship 2017;11:329-38.

13. Souza Mello V. Peroxisome proliferator-activated receptors as targets to treat non-alcoholic fatty liver disease. World J Hepatol 2015;7:1012.

14. Zafar SY, McNeil RB, Thomas CM, Lathan CS, Ayanian JZ, Provenzale D. Population-based assessment of cancer survivors' financial burden and quality of life: a prospective cohort study. J Oncol Practice 2015;11:145-50.

15. Loeb S, Bruinsma SM, Nicholson J, Briganti A, Pickles T, Kakehi $\mathrm{Y}$, et al. Active surveillance for prostate cancer: a systematic review of clinicopathologic variables and biomarkers for risk stratification. European Urol 2015;67:619-26.

16. Raszeja Wyszomirska J, Wunsch E, Krawczyk M, Rigopoulou EI, Kostrzewa K, Norman GL, et al. Assessment of health-related quality of life in polish patients with primary biliary cirrhosis. Clin Res Hepatol Gastroenterol 2016;40:471-9.

17. Schramm C, Wahl I, Weiler Normann C, Voigt K, Wiegard C, Glaubke C, et al. Health-related quality of life, depression, and anxiety in patients with autoimmune hepatitis. J Hepatol 2014;60:618-24.

18. Tana MM, Alao H, Morris N, Bernstein S, Hattenbach J, Rehman $\mathrm{RB}$, et al. Fatigued patients with chronic liver disease have subtle aberrations of sleep, melatonin and cortisol circadian rhythms. Fatigue: Biomed Health Behavior 2018;6:5-19.

19. Chugh Y, Dhiman RK, Premkumar M, Prinja S, Singh Grover G, Bahuguna P. Real-world cost-effectiveness of pan-genotypic sofosbuvir-velpatasvir combination versus genotypedependent directly acting anti-viral drugs for treatment of hepatitis $\mathrm{C}$ patients in the universal coverage scheme of punjab state in India. PloS One 2019;14:e0221769.

20. Dogar I, Siddiqui N, Bajwa A, Bhatti A, Haider N, Hashmi ZY. Relationship between liver diseases and levels of anxiety and depression. J Pak Psych Soc 2009;6:61-4.

21. Weinstein AA, Price JK, Stepanova M, Poms LW, Fang Y, Moon J, et al. Depression in patients with nonalcoholic fatty liver disease and chronic viral hepatitis B and C. Psychosomatics 2011;52:127-32.

22. Cron D, Friedman J, Winder G, Thelen A, Derck J, Fakhoury J, et al. Depression and frailty in patients with-semgle liver disease referred for transplant evaluation. Am J Transplant 2016;16:1805-11.

23. Perng CL, Shen CC, Hu LY, Yeh CM, Chen MH, Tsai CF, et al. Risk of depressive disorder following non-alcoholic cirrhosis: a nationwide population-based study. PloS One 2014;9:e88721.

24. Popovic DD, Culafic DM, Kisic Tepavcevic DB, Kovacevic NV, Spuran MM, Đuranovic SP, et al. Assessment of depression and anxiety in patients with chronic liver disease. Vojnosanitetski Pregled 2015;72:414-20.

25. Silva MJ, Rosa MV, Nogueira PJ, Calinas F. Ten years of hospital admissions for liver cirrhosis in portugal. Eur J Gastroenterol Hepatol 2015;27:1320-6. 
26. Zhu HP, Gu YR, Zhang GL, Su YJ, Wang K, Zheng YB, et al. Depression in patients with chronic hepatitis $\mathrm{B}$ and cirrhosis is closely associated with the severity of liver cirrhosis. Exp Ther Med 2016;12:405-9. 